\title{
Radiative pion capture by a nucleon
}

\author{
Harold W. Fearing, ${ }^{1, *}$ Thomas R. Hemmert, ${ }^{2, \dagger}$ Randy Lewis, ${ }^{3, \dagger}$ and Christine Unkmeir ${ }^{4, \S}$ \\ ${ }^{1}$ TRIUMF, 4004 Wesbrook Mall, Vancouver, British Columbia, Canada V6T 2A3 \\ ${ }^{2}$ Forschungszentrum Jülich, Institut für Kernphysik (Th), D-52425 Jülich, Germany \\ ${ }^{3}$ Department of Physics, University of Regina, Regina, Saskatchewan, Canada S4S OA2 \\ ${ }^{4}$ Institut für Kernphysik, Johannes Gutenberg-Universität, D-55099 Mainz, Germany
}

(Received 22 May 2000; published 20 October 2000)

\begin{abstract}
The differential cross sections for $\pi^{-} p \rightarrow \gamma n$ and $\pi^{+} n \rightarrow \gamma p$ are computed up to $O\left(p^{3}\right)$ in heavy baryon chiral perturbation theory (HBChPT). The expressions at $O(p)$ and $O\left(p^{2}\right)$ have no free parameters. There are three unknown parameters at $O\left(p^{3}\right)$, low energy constants of the HBChPT Lagrangian, which are determined by fitting to experimental data. Two acceptable fits are obtained, which can be separated by comparing with earlier dispersion relation calculations of the inverse process. Expressions for the multipoles, with emphasis on the $p$-wave multipoles, are obtained and evaluated at threshold. Generally the results obtained from the best of the two fits are in good agreement with the dispersion relation predictions.
\end{abstract}

PACS number(s): 12.39.Fe, 13.40.-f, 13.75.Gx

\section{INTRODUCTION}

Radiative pion capture by a nucleon is one of the obvious reactions to use as a testbed for heavy baryon chiral perturbation theory (HBChPT). For charged pions, the reaction begins at $O(p)$, which is leading order in HBChPT, and it is known that the $O\left(p^{3}\right)$ result for the $s$-wave multipole is in reasonable agreement with most measurements [1]. The $p$-wave multipoles, however, seem never to have been calculated. This is in contrast to the neutral pion case where both $s$ - and $p$-wave multipoles have been extensively discussed [2]. A calculation beyond the $s$ wave provides insight into the convergence of the chiral expansion and also serves to determine some of the HBChPT parameters that are required for other reactions, such as radiative muon capture by a nucleon, where the existing experimental data are in surprising disagreement with theoretical expectations [3]. Thus an investigation of the $p$-wave multipoles in the charged case is a useful thing to do and is the primary aim of this work.

In the present work, the only explicit fields in the chiral Lagrangian are the pions and nucleons. Other physical particles will enter the calculation through their implicit contributions to the Lagrangian's parameters (LEC's). For some reactions it is advantageous to include the $\Delta(1232)$ explicitly, as done for example in Ref. [4], and it is possible that this could be a useful approach for radiative pion capture as well, once one goes away from threshold. However, it is consistent to absorb such resonances into the LEC's and we shall see that for the present reaction a reasonable fit to the data can be obtained when the $\Delta(1232)$ is left implicit in the HBChPT parameters.

Experimental data for the $\pi^{-} p \rightarrow \gamma n$ differential cross section was reported 15 years ago from a TRIUMF experiment at beam energies of $T_{\pi}=27.4$ and $39.3 \mathrm{MeV}$ [5]. A

\footnotetext{
*Electronic address: fearing@triumf.ca

†Electronic address: th.hemmert@fz-juelich.de

\$Electronic address: randy.lewis@ uregina.ca

${ }^{\S}$ Electronic address: unkmeir@kph.uni-mainz.de
}

recent TRIUMF experiment has taken data at $T_{\pi}=9.88$, 14.62, and $19.85 \mathrm{MeV}$ [6]. There are also very recent data [7] for the inverse reaction $\gamma p \rightarrow n \pi^{+}$taken very near threshold at $T_{\gamma} \simeq 153 \mathrm{MeV}$ corresponding to $T_{\pi} \simeq 3 \mathrm{MeV}$. In this study, we will not attempt to apply HBChPT when the pion energy is greater than $40 \mathrm{MeV}$.

There are at least two modern theoretical discussions of radiative charged-pion capture (both discussions actually address the inverse reaction: charged-pion photoproduction). One is an HBChPT study of the $s$ wave at threshold by Bernard, Kaiser, and Meißner [1], and another is a dispersion theoretical analysis of $s$ and $p$ waves by Hanstein, Drechsel, and Tiator [8]. The present work goes beyond threshold and also explicitly computes the $p$-wave multipoles. The comparison of our work to the threshold results of Ref. [8] is found to be quite interesting and to provide a useful constraint on our results.

In Sec. II, we establish the general expressions for kinematics, multipoles and the differential cross section. Section III discusses the HBChPT calculation and Sec. IV presents and discusses our results, both at threshold and in general. Section V contains a summary of what has been learned from this effort, and what the next steps could be.

\section{KINEMATICS AND MULTIPOLES}

In radiative charged-pion capture by a nucleon, a low en$\operatorname{ergy} \pi^{ \pm}$with four-momentum $q^{\mu}=\left(E_{\pi}, \vec{q}\right)$ in the center-ofmass system gets absorbed by a slowly moving nucleon of mass $m_{N}$. In the final state, one observes a recoiling nucleon and a low energy photon with polarization four-vector $\epsilon^{\mu}$ $=\left(\boldsymbol{\epsilon}_{0}, \overrightarrow{\boldsymbol{\epsilon}}\right)$ and four-momentum $k^{\mu}=(\omega, \vec{k})$. The pion's center-of-mass energy is related to $s$, the square of the total energy in the center of mass, and to $T_{\pi}$, the kinetic energy in the lab frame by

$$
E_{\pi}=\frac{s+m_{\pi}^{2}-m_{N}^{2}}{2 \sqrt{s}}=\frac{m_{\pi}^{2}+m_{N}\left(m_{\pi}+T_{\pi}\right)}{\sqrt{\left(m_{N}+m_{\pi}\right)^{2}+2 m_{N} T_{\pi}}},
$$


where $m_{\pi}$ and $m_{N}$ are, respectively, the pion and nucleon masses. The analogous formulas for the photon energy in the center of mass are

$$
\omega=\frac{s-m_{N}^{2}}{2 \sqrt{s}}=\frac{m_{N} T_{\gamma}}{\sqrt{m_{N}^{2}+2 m_{N} T_{\gamma}}},
$$

where $T_{\gamma}$ is the corresponding laboratory $\gamma$ energy for the inverse process. All energy dependence will be expressed via the pion energy in the center-of-mass system. For the energy of the final state photon we therefore employ

$$
\omega=E_{\pi}-\frac{m_{\pi}^{2}}{2 m_{N}}+\frac{E_{\pi} m_{\pi}^{2}}{2 m_{N}^{2}}+\mathcal{O}\left(1 / m_{N}^{3}\right) .
$$

The differential cross section for the pion capture process in the center-of-mass frame is

$$
\frac{d \sigma^{\pi N \rightarrow \gamma N}}{d \Omega_{\gamma}}=\frac{\omega}{|\vec{q}|} \frac{1}{2} \sum_{\text {pols }}|\mathcal{M}|^{2},
$$

and that for the inverse (photoproduction) reaction is

$$
\frac{d \sigma^{\gamma N \rightarrow \pi N}}{d \Omega_{\pi}}=\frac{|\vec{q}|}{\omega} \frac{1}{4} \sum_{\text {pols }}|\mathcal{M}|^{2},
$$

where $\mathcal{M}$ is the amplitude defined below. Notice that Eqs. (4) and (5) explicitly contain the average over initial and sum over final spins and polarizations and that the two cross sections are related by the usual detailed balance relation.

Essentially all previous work has dealt with the inverse, photoproduction, process, $\gamma N \rightarrow \pi N$ and the conventions for that process are by now well established. Thus in the Coulomb gauge with $\epsilon_{0}=0$ and the transversality condition $\vec{\epsilon} \cdot \vec{k}=0$ the amplitude for that process can be written in terms of the $T$ matrix as $[9,10]$

$$
\begin{aligned}
\mathcal{M}^{\gamma N \rightarrow \pi N}= & \frac{m_{N}}{4 \pi \sqrt{s}} T \cdot \epsilon \\
= & F_{1}\left(E_{\pi}, x\right) i \chi^{\dagger} \vec{\sigma} \cdot \vec{\epsilon} \chi+F_{2}\left(E_{\pi}, x\right) \chi^{\dagger} \vec{\sigma} \cdot \hat{q} \\
& \times \vec{\sigma} \cdot(\hat{k} \times \vec{\epsilon}) \chi+F_{3}\left(E_{\pi}, x\right) i \chi^{\dagger} \vec{\sigma} \cdot \hat{k} \vec{\epsilon} \cdot \hat{q} \chi \\
& +F_{4}\left(E_{\pi}, x\right) i \chi^{\dagger} \vec{\sigma} \cdot \hat{q} \vec{\epsilon} \cdot \hat{q} \chi,
\end{aligned}
$$

where $\sigma^{i}$ is a Pauli matrix in spin space between the twocomponent spinors of the incoming/outgoing nucleon $\left(\chi / \chi^{\dagger}\right), \epsilon$ is the photon polarization vector and $x=\cos \theta$ corresponds to the cosine of the angle between the photon and the pion momenta.

Furthermore, each structure amplitude $F_{i}\left(E_{\pi}, x\right)$, $(i=1,2,3,4)$, can be decomposed into three isospin channels $(a=1,2,3)$

$$
\begin{aligned}
F_{i}^{a}\left(E_{\pi}, x\right)= & F_{i}^{(-)}\left(E_{\pi}, x\right) i \epsilon^{a 3 b} \tau^{b}+F_{i}^{(0)}\left(E_{\pi}, x\right) \tau^{a} \\
& +F_{i}^{(+)}\left(E_{\pi}, x\right) \delta^{a 3}
\end{aligned}
$$

where $\tau^{a}$ denotes a Pauli matrix in isospin space. The physical structure amplitudes are then obtained from the linear combinations

$$
\begin{aligned}
& F_{i}^{\gamma n \rightarrow \pi^{-} p}=\sqrt{2}\left[F_{i}^{(0)}-F_{i}^{(-)}\right], \\
& F_{i}^{\gamma p \rightarrow \pi^{+} n}=\sqrt{2}\left[F_{i}^{(0)}+F_{i}^{(-)}\right] .
\end{aligned}
$$

The full physics content of this process is encoded in the four structure amplitudes $F_{i}$, which are complicated functions of $E_{\pi}$ and $\theta$, and in the amplitude of Eq. (6), the square of which is used to get the cross section. However it may be more intuitive to discuss the underlying physics in terms of a multipole decomposition. The HBChPT formalism which we are employing in the following sections involves an expansion in terms of the pion energy divided by a scale of approximately $1 \mathrm{GeV}$, i.e., it is only reliable in a kinematic region of low energy pions. With this in mind we restrict the multipoles we consider to $s$ and $p$ waves only. They can be found from the $F$ amplitudes via $[9,10]$

$$
\begin{aligned}
E_{0+}\left(E_{\pi}\right)= & \int_{-1}^{1} d x\left\{\frac{1}{2} F_{1}\left(E_{\pi}, x\right)-\frac{1}{2} x F_{2}\left(E_{\pi}, x\right)\right. \\
& \left.+\frac{1}{6}\left[1-P_{2}(x)\right] F_{4}\left(E_{\pi}, x\right)\right\}, \\
M_{1+}\left(E_{\pi}\right)= & \int_{-1}^{1} d x\left\{\frac{1}{4} x F_{1}\left(E_{\pi}, x\right)-\frac{1}{4} P_{2}(x) F_{2}\left(E_{\pi}, x\right)\right. \\
+ & \left.\frac{1}{12}\left[P_{2}(x)-1\right] F_{3}\left(E_{\pi}, x\right)\right\}, \\
M_{1-}\left(E_{\pi}\right)= & \int_{-1}^{1} d x\left\{-\frac{1}{2} x F_{1}\left(E_{\pi}, x\right)+\frac{1}{2} F_{2}\left(E_{\pi}, x\right)\right. \\
& \left.+\frac{1}{6}\left[1-P_{2}(x)\right] F_{3}\left(E_{\pi}, x\right)\right\}, \\
+ & \left.\frac{1}{10}\left[x-P_{3}(x)\right] F_{4}\left(E_{\pi}, x\right)\right\} \\
+ & \frac{1}{12}\left[1-P_{2}(x)\right] F_{3}\left(E_{\pi}, x\right) \\
E_{1+}\left(E_{\pi}\right)= & d x\left\{\frac{1}{4} x F_{1}\left(E_{\pi}, x\right)-\frac{1}{4} P_{2}(x) F_{2}\left(E_{\pi}, x\right)\right. \\
& \left.\int_{-1}\right)
\end{aligned}
$$

with the $P_{i}(x), i \geqslant 2$ being Legendre polynomials.

The formulas above are those conventionally defined for the photoproduction reaction $\gamma N \rightarrow \pi N$, whereas we are interested particularly in the capture process $\pi N \rightarrow \gamma N$. The cross sections for these two processes are related trivially by the detailed balance equation arising from Eqs. (4) and (5). The relation between the amplitudes is however more complicated, arising from time reversal and depending explicitly on the phases of the parts of the amplitude. In our conventions we find (up to a possible overall, and thus irrelevant phase) 


$$
\mathcal{M}^{\pi N \rightarrow \gamma N}=-\left[\mathcal{M}^{\gamma N \rightarrow \pi N}\right]^{*} .
$$

If we apply Eq. (14) to Eq. (6) to get the amplitude for pion capture the structure functions $F_{i}$ attract various phases and a complex conjugate and the order of the structures corresponding to $F_{2}$ is reversed. Putting the $F_{2}$ structures back in the original order generates extra terms and makes some of the coefficients of the four independent structures linear combinations of the $F_{i}$. Thus if we were to define the amplitude for the pion capture reaction to be of the original general form of Eq. (6) then the $F_{i}$ for pion capture will be linear combinations, complex conjugated, with various phase changes, of the $F_{i}$ for photoproduction. An alternative, and probably more sensible choice, is to define the amplitude for the capture reaction via the action of Eq. (14) on the definition used for the photoproduction direction. This eliminates the problem of linear combinations, but still leaves the two sets of $F_{i}$ related by a complex conjugate and various phase changes.

The third alternative, which is the one we adopt, is to just do the calculation for the photoproduction direction in the first place, and then make the connection to the pion capture direction at the level of the cross section. This has the advantage of keeping a close connection with the conventions and the large body of previous work dealing with photoproduction. Thus the formulas for the $F_{i}$ which we quote, and more importantly those for the multipoles, are actually for the $\gamma N \rightarrow \pi N$ direction. This means, for example, that our numerical results for the multipoles can be compared directly and without ambiguity with the dispersion relation calculation for photoproduction of Ref. [8], even though the parameters are being fixed primarily by the pion capture data.

\section{THE HBChPT CALCULATION}

The HBChPT Lagrangian is ordered in powers of momenta and pion masses, which are small compared to both the chiral scale, $4 \pi F$, and the nucleon mass $m_{N}$

$$
\mathcal{L}_{\pi N}=\mathcal{L}_{\pi N}^{(1)}+\mathcal{L}_{\pi N}^{(2)}+\mathcal{L}_{\pi N}^{(3)}+\cdots
$$

The lowest-order Lagrangian is

$$
\mathcal{L}_{\pi N}^{(1)}=\bar{N}_{v}\left(i v \cdot \nabla+g_{A} S \cdot u\right) N_{v},
$$

where $[11,12]$

$$
\begin{gathered}
N_{v}(x)=\exp \left[i m_{0 N} v \cdot x\right] \frac{1}{2}(1+v) \psi(x), \\
S_{\mu}=\frac{i}{2} \gamma_{5} \sigma_{\mu \nu} v^{\nu}, \\
u_{\mu}=i u^{\dagger}\left(\partial_{\mu}-i r_{\mu}\right) u-i u\left(\partial_{\mu}-i l_{\mu}\right) u^{\dagger}, \\
\nabla_{\mu}=\partial_{\mu}+\Gamma_{\mu}-i v_{\mu}^{(s)}, \\
\Gamma_{\mu}=\frac{1}{2}\left[u^{\dagger}\left(\partial_{\mu}-i r_{\mu}\right) u+u\left(\partial_{\mu}-i l_{\mu}\right) u^{\dagger}\right],
\end{gathered}
$$

with $m_{0 N}$ and $g_{A}$ being the lowest-order nucleon mass and axial coupling, respectively. The external photon field is included via $r_{\mu}=l_{\mu}=-(e / 2) \tau^{3} A_{\mu}$, and $u$ is a nonlinear representation of the pion fields, for example,

$$
u=\exp \left[\frac{i}{2 F_{0}}\left(\begin{array}{cc}
\pi^{0} & \sqrt{2} \pi^{+} \\
\sqrt{2} \pi^{-} & -\pi^{0}
\end{array}\right)\right] .
$$

The parameter $F_{0}$ corresponds to the pion decay constant in the chiral limit (normalized so that the physical value $F$ $=92.4 \mathrm{MeV}$ ).

The higher-order Lagrangians $\mathcal{L}_{\pi N}^{(n)}$ will be written in the notation of Ecker and Mojžis [11] and are exactly the same as those used in Ref. [12]. Results for the multipoles in the present work depend on four combinations of parameters from $\mathcal{L}_{\pi N}^{(3)}$, namely $b_{10}, b_{19}, b_{21}^{r}(\mu)$, and $2 b_{22}^{r}(\mu)+b_{23}$, where $\mu$ is the renormalization scale. The numerical values of $b_{19}$ and $b_{23}$ were determined in Ref. [12]. The three remaining parameters $b_{10}, b_{21}^{r}(\mu)$, and $b_{22}^{r}(\mu)$, will be determined in the present work.

The calculation requires an evaluation of tree-level and one-pion-loop diagrams, which can be organized into four classes depending on whether the radiated photon is emitted from the initial nucleon, the final nucleon, the pion, or from the $\pi N N$ vertex. The calculation was performed in a general gauge (and is fully gauge invariant). While this meant more work, the ability to check gauge invariance provided a very important tool for eliminating errors in what was an algebraically complex calculation. The result was then reduced to the special case of $v \cdot \epsilon=0$. In this gauge, only one of the four classes of diagrams has any dependence on the unknown HBChPT parameters $b_{10}, b_{21}^{r}(\mu)$ and $b_{22}^{r}(\mu)$, namely, photon emission from the $\pi N N$ vertex.

Adding all contributions together gives the amplitude of Eq. (6) with the structure amplitudes, $F_{i}\left(E_{\pi}, x\right)$, given explicitly in the Appendix. Although only charged-pion processes are discussed in this work, the calculation was actually performed for general isospin. We have verified that the $\pi^{0}$ amplitudes agree with Ref. [2].

\section{RESULTS}

\section{A. The differential cross section}

Using our calculation from the previous section with Eqs. (4) or (5) and the $\mathcal{M}$ of Eq. (6) and the F's of the Appendix, we can immediately compute the differential cross section. At $O(p)$ and $O\left(p^{2}\right)$ the result is completely determined, whereas at $O\left(p^{3}\right)$ it depends on three unknown parameters, which will now be determined via a least-squares fit to the experimental data.

Reference [6] provides 11 measurements of the differential cross section for $\pi^{-} p \rightarrow \gamma n$ at $T_{\pi}=9.88,14.62$, and 19.85 MeV and Ref. [5] provides an additional 16 measure- 
TABLE I. Values of the three coefficients in the $O\left(p^{3}\right)$ HBChPT Lagrangian which are obtained from a least-squares, three parameter, fit to various subsets of the experimental data. In each case there are two roughly equivalent well defined minima of $\chi^{2}$ per degree of freedom labeled by A and B. The data consist of (a) 11 measurements of $\pi^{-} p \rightarrow \gamma n$ with $T_{\pi} \leqslant 19.85 \mathrm{MeV}$ [6]; (b) 16 measurements of $\pi^{-} p \rightarrow \gamma n$ with $T_{\pi}$ $\geqslant 27.4 \mathrm{MeV}$ [5]; and (c) eight measurements of $\gamma p \rightarrow n \pi^{+}$at $T_{\gamma} \simeq 153 \mathrm{MeV}$ [7]. The arguments of A and B correspond to the number of data in the set chosen so that $11 \sim \operatorname{set}(a), 16 \sim \operatorname{set}(b), 27 \sim \operatorname{sets}(\mathrm{a})+(\mathrm{b})$, and $35 \sim$ sets (a) + (b) + (c). As input, we use $b_{19}=-0.7 \pm 0.4$ and $b_{23}=-3.1 \pm 0.3$ as determined in Ref. [12]. Note that $b_{22}^{r}$ appears only in the combination $2 b_{22}^{r}+b_{23}$, so that its value obtained from fitting these data depends on the value taken for $b_{23}$. As discussed in the text, $\mathrm{A}(35)$ is considered to be the best result.

\begin{tabular}{ccccc}
\hline \hline & $\chi^{2} /$ d.o.f. & $b_{10}$ & $b_{21}^{r}\left(m_{N}\right)$ & $b_{22}^{r}\left(m_{N}\right)$ \\
\hline $\mathrm{A}(11)$ & 2.79 & $8.8 \pm 16.1$ & $-8.2 \pm 1.1$ & $9.2 \pm 1.1$ \\
$\mathrm{~A}(16)$ & 1.12 & $6.1 \pm 9.1$ & $-7.6 \pm 1.1$ & $9.3 \pm 0.8$ \\
$\mathrm{~A}(27)$ & 1.62 & $11.9 \pm 5.4$ & $-8.2 \pm 0.7$ & $9.3 \pm 0.6$ \\
$\mathrm{~A}(35)$ & 1.59 & $13.7 \pm 4.5$ & $-8.2 \pm 0.7$ & $9.2 \pm 0.6$ \\
$\mathrm{~B}(11)$ & 2.81 & $-40.5 \pm 15.7$ & $-8.2 \pm 1.1$ & $9.3 \pm 1.0$ \\
$\mathrm{~B}(16)$ & 1.15 & $-36.2 \pm 9.2$ & $-7.6 \pm 1.0$ & $9.4 \pm 0.8$ \\
$\mathrm{~B}(27)$ & 1.63 & $-42.4 \pm 5.3$ & $-8.3 \pm 0.7$ & $9.4 \pm 0.7$ \\
$\mathrm{~B}(35)$ & 1.67 & $-45.6 \pm 4.6$ & $-8.4 \pm 0.7$ & $9.4 \pm 0.7$ \\
\hline \hline
\end{tabular}

ments at $T_{\pi}=27.4$ and $39.3 \mathrm{MeV}$. A further eight measurements, these for the inverse reaction $\gamma p \rightarrow n \pi^{+}$very near threshold ( $T_{\pi} \simeq 3 \mathrm{MeV}$ ), come from Ref. [7]. We have performed fits to several subsets of this set of data, as well as to the complete set. A comparison of these fits allows us to check for consistency among the data sets and also for a possible breakdown of the HBChPT form as $T_{\pi}$ increases.

The values of the three fitted parameters are given in Table I. It is reassuring to see that within the uncertainties all of the various data sets lead to the same numerical values for these parameters, though the fit becomes more stable and the uncertainties smaller as we increase the number of data points included in the fit. It should also be noted that each of our least-squares fits actually finds two sets of parameters, characterized by nearly identical values of $b_{21}^{r}$ and $b_{22}^{r}$ but quite different values of $b_{10}$, depending on where the leastsquares routine begins in parameter space. This presumedly reflects the fact that the cross section is quadratic in the $b_{i}$ 's and that the data are not sufficiently good to distinguish the two solutions. We refer to these two minima in parameter space as $\mathrm{A}$ and $\mathrm{B}$, and then label our solution sets as $\mathrm{A}(n)$ and $\mathrm{B}(n)$, where $n$ is the number of experimental measurements used in the fit. For the various subsets of pion capture data, $\mathrm{A}(n)$ and $\mathrm{B}(n)$ give essentially indistinguishable $\chi^{2}$ values and differential cross sections. Addition of the very low energy photoproduction data of Ref. [7] produces a small improvement in the $\chi^{2}$ of the $\mathrm{A}(35)$ solution relative to the $\mathrm{B}(35)$ one. The two solutions can be distinguished, however, by their quite different values of $b_{10}$ and also by the different individual $p$-wave multipoles, as will be discussed below.

The results of our best fits to the cross section data are shown in Fig. 1, along with the parameter-free $O(p)$ and $O\left(p^{2}\right)$ calculations and the experimental data. As these plots indicate, the $O(p)$ calculation disagrees with the data. $O\left(p^{2}\right)$ contributions reduce the discrepancy, but do not eliminate it. The $O\left(p^{3}\right)$ terms are necessary for a good fit to the data. The $O(p)$ terms clearly dominate (note the suppressed zero in the plots), but the contributions of $O\left(p^{2}\right)$ and $O\left(p^{3}\right)$ are comparable at most angles. For $\gamma n \leftrightarrow \pi^{-} p$ the two contributions seem to add, whereas for $\gamma p \leftrightarrow \pi^{+} n$ they have opposite signs and tend to cancel. The fact that the $O\left(p^{2}\right)$ and $O\left(p^{3}\right)$ terms are more or less equal may raise some concern that the HBChPT expansion has not yet fully converged at $O\left(p^{3}\right)$. This point can also be made from Table I, which gives the values of the three parameters that were determined in the fits. For a nicely converging chiral expansion that just contains pions and nucleons as effective degrees of freedom, one probably would have expected each of the $b_{i}$ to acquire values near unity. The fact that we find values somewhat larger than this perhaps can be seen as an indication of the role of explicit matter fields like the $\Delta$ isobar and vector mesons. The discussion of such issues, however, has to be delayed to a future communication. Here we only provide the first step and fix the contact terms numerically at the scale $\mu=m_{N}$. Note, however, that the value of $b_{10}$ obtained from the A (but not the B) solution is quite consistent in magnitude with the value of the parameter $b_{P}$, which is a linear combination of $b_{10}$ and $b_{9}$, obtained in Ref. [2] by fitting $\pi^{0}$ data.

\section{B. Threshold results}

Expressions are simplified somewhat at threshold, that is, in the limit in which the pion kinetic energy $T_{\pi}$ goes to zero. Using $\quad M_{1+}=\omega|\vec{q}| m_{1+}, M_{1-}=\omega|\vec{q}| m_{1-}, \quad$ and $E_{1+}$ $=\omega|\vec{q}| e_{1+}$, the multipoles, given for the photoproduction process $\gamma N \rightarrow \pi N$, follow directly from Eqs. (10)-(13) and the expressions for the $F$ 's given in the Appendix. The resulting expressions are given purely in terms of physical quantities. For the $(0)$ isospin channel we obtain

$$
E_{0+}^{(0)}\left(m_{\pi}\right)=\frac{m_{N}}{4 \pi\left(m_{N}+m_{\pi}\right)} \frac{e G_{A}}{2 F}\left[-\frac{m_{\pi}}{2 m_{N}}+\frac{m_{\pi}^{2}}{4 m_{N}^{2}}\left(\mu_{p}+\mu_{n}\right)\right],
$$



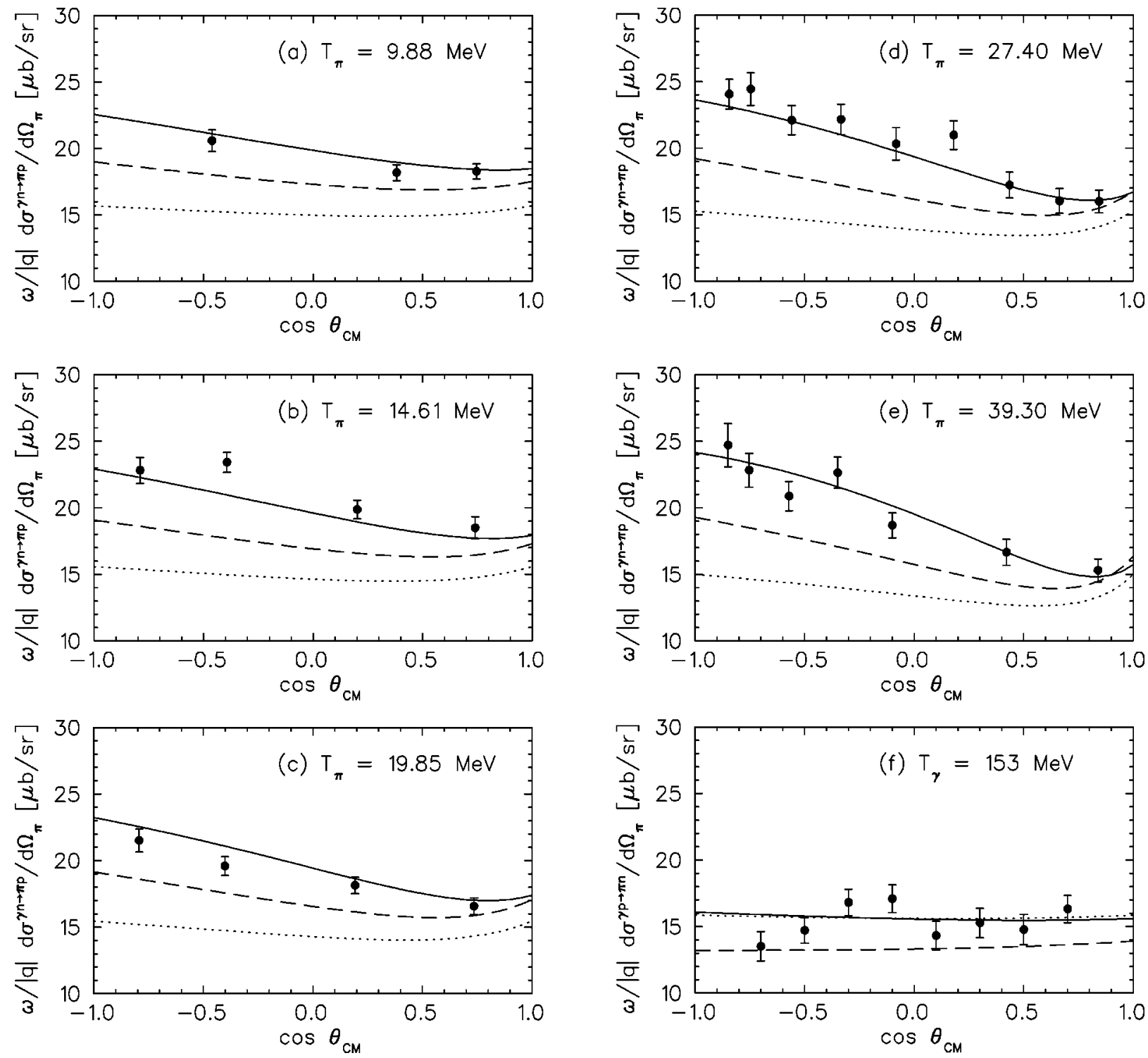

FIG. 1. The cross section for the pion capture reaction, quoted as the reduced center-of-mass cross section for the inverse $\gamma n \rightarrow \pi^{-} p$ or [for (f)] $\gamma p \rightarrow \pi^{+} n$ reaction. Experimental data are compared to the HBChPT predictions at $O(p)$ (dotted line), $O\left(p^{2}\right)$ (dashed line), and $O\left(p^{3}\right)$ (solid line). The $O\left(p^{3}\right)$ result corresponds to $\mathrm{A}(35)$ and $\mathrm{B}(35)$ which are indistinguishable in these plots. (a) $T_{\pi}=9.88 \mathrm{MeV}$, (b) $T_{\pi}=14.62 \mathrm{MeV}$, (c) $T_{\pi}=19.85 \mathrm{MeV}$, (d) $T_{\pi}=27.4 \mathrm{MeV}$, (e) $T_{\pi}=39.3 \mathrm{MeV}$, (f) $T_{\gamma}=153 \mathrm{MeV}, T_{\pi}=3.06 \mathrm{MeV}$.

$$
\begin{gathered}
m_{1+}^{(0)}\left(m_{\pi}\right)=\frac{m_{N}}{4 \pi\left(m_{N}+m_{\pi}\right)} \frac{e G_{A}}{2 F}\left[\frac{\left(\mu_{p}+\mu_{n}\right)}{6 m_{\pi} m_{N}}-\frac{1}{12 m_{N}^{2}}+\frac{\left(\mu_{p}+\mu_{n}\right)}{6 m_{N}^{2}}+\frac{2 b_{10}}{3 G_{A}(4 \pi F)^{2}}\right], \\
m_{1-}^{(0)}\left(m_{\pi}\right)=\frac{m_{N}}{4 \pi\left(m_{N}+m_{\pi}\right)} \frac{e G_{A}}{2 F}\left[-\frac{\left(\mu_{p}+\mu_{n}\right)}{3 m_{\pi} m_{N}}+\frac{7}{24 m_{N}^{2}}-\frac{\left(\mu_{p}+\mu_{n}\right)}{3 m_{N}^{2}}+\frac{2 b_{10}}{3 G_{A}(4 \pi F)^{2}}\right], \\
e_{1+}^{(0)}\left(m_{\pi}\right)=\frac{m_{N}}{4 \pi\left(m_{N}+m_{\pi}\right)} \frac{e G_{A}}{2 F} \frac{1}{24 m_{N}^{2}},
\end{gathered}
$$

and for the (-) isospin channel 


$$
\begin{aligned}
& E_{0+}^{(-)}\left(m_{\pi}\right)=\frac{m_{N}}{4 \pi\left(m_{N}+m_{\pi}\right)} \frac{e G_{A}}{2 F}\left[1+\frac{m_{\pi}^{2}}{8 m_{N}^{2}}-\frac{m_{\pi}^{2}}{4 m_{N}^{2}}\left(\mu_{p}-\mu_{n}\right)+\frac{\pi^{2} m_{\pi}^{2}}{4(4 \pi F)^{2}}\right. \\
& \left.-\frac{m_{\pi}^{2}}{G_{A}(4 \pi F)^{2}}\left(2 b_{19}-2 b_{21}^{r}(\mu)-2 b_{22}^{r}(\mu)-b_{23}+G_{A} \ln \frac{m_{\pi}^{2}}{\mu^{2}}\right)\right] \\
& m_{1+}^{(-)}\left(m_{\pi}\right)=\frac{m_{N}}{4 \pi\left(m_{N}+m_{\pi}\right)} \frac{e G_{A}}{2 F}\left[-\frac{1}{6 m_{\pi}^{2}}-\frac{1}{12 m_{\pi} m_{N}}-\frac{\left(\mu_{p}-\mu_{n}\right)}{6 m_{\pi} m_{N}}+\frac{5}{48 m_{N}^{2}}-\frac{\left(\mu_{p}-\mu_{n}\right)}{6 m_{N}^{2}}+\frac{2 G_{A}^{2}}{3(4 \pi F)^{2}}\right. \\
& \left.-\frac{2 G_{A}^{2} \pi}{3(4 \pi F)^{2}}+\frac{G_{A}^{2} \pi^{2}}{12(4 \pi F)^{2}}+\frac{1}{6 G_{A}(4 \pi F)^{2}}\left(2 b_{19}-4 b_{22}^{r}(\mu)-2 b_{23}-2 G_{A}^{3} \ln \frac{m_{\pi}^{2}}{\mu^{2}}\right)\right] \\
& m_{1-}^{(-)}\left(m_{\pi}\right)=\frac{m_{N}}{4 \pi\left(m_{N}+m_{\pi}\right)} \frac{e G_{A}}{2 F}\left[\frac{1}{3 m_{\pi}^{2}}+\frac{1}{6 m_{\pi} m_{N}}-\frac{\left(\mu_{p}-\mu_{n}\right)}{6 m_{\pi} m_{N}}+\frac{1}{24 m_{N}^{2}}-\frac{\left(\mu_{p}-\mu_{n}\right)}{6 m_{N}^{2}}-\frac{4 G_{A}^{2}}{3(4 \pi F)^{2}}-\frac{2 G_{A}^{2} \pi}{3(4 \pi F)^{2}}\right. \\
& \left.+\frac{G_{A}^{2} \pi^{2}}{3(4 \pi F)^{2}}-\frac{1}{3 G_{A}(4 \pi F)^{2}}\left(2 b_{19}-4 b_{22}^{r}(\mu)-2 b_{23}-2 G_{A}^{3} \ln \frac{m_{\pi}^{2}}{\mu^{2}}\right)\right], \\
& e_{1+}^{(-)}\left(m_{\pi}\right)=\frac{m_{N}}{4 \pi\left(m_{N}+m_{\pi}\right)} \frac{e G_{A}}{2 F}\left[\frac{1}{6 m_{\pi}^{2}}+\frac{1}{12 m_{\pi} m_{N}}-\frac{5}{48 m_{N}^{2}}-\frac{b_{19}}{3 G_{A}(4 \pi F)^{2}}\right] .
\end{aligned}
$$

To make contact with previous work, observe that the $O(p)$ and $O\left(p^{2}\right)$ parts of these expressions are just what one would obtain from an expansion of the usual Born graphs using pseudovector coupling. The $O\left(p^{3}\right)$ parts contain higher order pieces of the expansion of the Born graphs, loop contributions, and contributions from the part of the Lagrangian involving the LEC's.

The numerical values of the threshold multipoles at each order in HBChPT are displayed in Table II. The $O\left(p^{3}\right)$ results are given for both solutions $\mathrm{A}(n)$ and $\mathrm{B}(n)$. Again the results are essentially the same within errors for any of the subsets of data used, though the fit is most accurate when the full 35 points are included. The $m_{1+}$ and $m_{1-}$ multipoles differ dramatically between $\mathrm{A}(n)$ and $\mathrm{B}(n)$, as they have an important dependence on $b_{10}$ which is quite different for the two solutions. $e_{1+}$ is constant, as it depends only on the parameter $b_{19}$ which was fixed from muon capture [12] and $E_{0+}$ is nearly constant as it depends only on the parameters $b_{22}^{r}, b_{21}^{r}, b_{19}$, and $b_{23}$ which are all essentially the same for the two fits.

Also shown in Table II are the results of a dispersion theory calculation by Hanstein, Drechsel, and Tiator [8]. For the electric multipoles $E_{0+}$ and $e_{1+}$ the agreement with the HBChPT results is quite good for both the $\pi^{+}$and $\pi^{-}$cases. For the magnetic multipoles $m_{1+}$ and $m_{1-}$ the agreement with $\mathrm{A}(35)$ is good, albeit not spectacular. One must recognize, however, that there are uncertainties in the dispersion relations results also, which were quoted only for the $E_{0+}$ multipole. For the B(35) fit, however, the HBChPT and dispersion results for these multipoles are quite different. Thus comparison with the dispersion relation results strongly favors the $\mathrm{A}(35)$ solution over the $\mathrm{B}(35)$ one.

One can gain some further insight via a more detailed comparison with the dispersion relation results. Observe first that Eqs. (23) - (30) give the eight observable multipole amplitudes in terms of four parameters $b_{10}, b_{19}, b_{21}^{r}(\mu)$, and $2 b_{22}^{r}(\mu)+b_{23}$. This means that four parameter-free relations exist among the multipoles in the $O\left(p^{3}\right)$ HBChPT calculation. For example, Table III gives a set of four quantities which are independent of these four parameters, along with their values as obtained from HBChPT and dispersion theory. For these four quantities the convergence of the HBChPT expansion is good and the results agree quite well with the dispersion relation predictions of Ref. [8].

This idea can be carried a step further by looking at combinations of the multipoles which depend on only one or only a few of the $b_{i}$ 's. Such results are tabulated in Table IV. The multipole $e_{1+}^{(-)}$depends, in fact only weakly, on $b_{19}$ and one can see from the table that the HBChPT results converge well and agree with the dispersion theory result. The next two entries $m_{1+}^{(-)}$and $m_{1-}^{(-)}$depend in addition on the combination $2 b_{22}^{r}+b_{23}$ and also resemble the dispersion relation results. The next entry $E_{0+}^{(-)}$depends in addition on $b_{21}^{r}$ and the following one, $E_{0+}^{(-)}+3 m_{\pi}^{2}\left(m_{1+}^{(-)}-e_{1+}^{(-)}\right)$depends only on $b_{21}^{r}$. Both show good convergence and reasonable agreement with the dispersion theory. Finally the last two entries $m_{1+}^{(0)}$ 
TABLE II. Threshold $s$ - and $p$-wave multipoles for the reactions $\gamma n \rightarrow \pi^{-} p$ and $\gamma p \rightarrow \pi^{+} n$. The $E_{0+}$ are in units of $10^{-3} / m_{\pi^{+}}$and the reduced $p$-wave multipoles are in units of $10^{-3} / m_{\pi^{+}}^{3}$. Dispersion theory results are quoted from Ref. [8].

\begin{tabular}{|c|c|c|c|c|c|c|c|}
\hline & & & & $E_{0+}$ & $m_{1+}$ & $m_{1-}$ & $e_{1+}$ \\
\hline \multirow[t]{11}{*}{$\gamma n \rightarrow \pi^{-} p$} & HBChPT & $O(p)$ & & -28.2 & 4.7 & -9.4 & -4.7 \\
\hline & & $O\left(p^{2}\right)$ & & -30.3 & 9.0 & -8.1 & -5.1 \\
\hline & & $O\left(p^{3}\right)$ & $\mathrm{A}(11)$ & $-32.2 \pm 1.0$ & $12.6 \pm 3.5$ & $-7.4 \pm 3.5$ & $-5.04 \pm 0.04$ \\
\hline & & $O\left(p^{3}\right)$ & $\mathrm{A}(16)$ & $-32.7 \pm 0.9$ & $12.1 \pm 2.0$ & $-8.0 \pm 2.0$ & $-5.04 \pm 0.04$ \\
\hline & & $O\left(p^{3}\right)$ & $\mathrm{A}(27)$ & $-32.3 \pm 0.7$ & $13.3 \pm 1.2$ & $-6.8 \pm 1.2$ & $-5.04 \pm 0.04$ \\
\hline & & $O\left(p^{3}\right)$ & $\mathrm{A}(35)$ & $-32.2 \pm 0.7$ & $13.7 \pm 1.0$ & $-6.4 \pm 1.0$ & $-5.04 \pm 0.04$ \\
\hline & & $O\left(p^{3}\right)$ & $\mathrm{B}(11)$ & $-32.3 \pm 1.0$ & $2.0 \pm 3.4$ & $-18.1 \pm 3.4$ & $-5.04 \pm 0.04$ \\
\hline & & $O\left(p^{3}\right)$ & $\mathrm{B}(16)$ & $-32.7 \pm 0.9$ & $2.9 \pm 2.0$ & $-17.2 \pm 2.0$ & $-5.04 \pm 0.04$ \\
\hline & & $O\left(p^{3}\right)$ & $\mathrm{B}(27)$ & $-32.3 \pm 0.7$ & $1.6 \pm 1.2$ & $-18.6 \pm 1.2$ & $-5.04 \pm 0.04$ \\
\hline & & $O\left(p^{3}\right)$ & $\mathrm{B}(35)$ & $-32.2 \pm 0.7$ & $0.9 \pm 1.0$ & $-19.2 \pm 1.0$ & $-5.04 \pm 0.04$ \\
\hline & Dispersion theory & & & $-31.7 \pm 0.2$ & 11.2 & -8.3 & -4.9 \\
\hline \multirow[t]{11}{*}{$\gamma p \rightarrow \pi^{+} n$} & HBChPT & $O(p)$ & & 28.2 & -4.7 & 9.4 & 4.7 \\
\hline & & $O\left(p^{2}\right)$ & & 26.1 & -7.7 & 5.6 & 5.1 \\
\hline & & $O\left(p^{3}\right)$ & $\mathrm{A}(11)$ & $28.3 \pm 1.0$ & $-7.5 \pm 3.5$ & $8.8 \pm 3.5$ & $5.09 \pm 0.04$ \\
\hline & & $O\left(p^{3}\right)$ & $\mathrm{A}(16)$ & $28.8 \pm 0.9$ & $-8.1 \pm 2.0$ & $8.2 \pm 2.0$ & $5.09 \pm 0.04$ \\
\hline & & $O\left(p^{3}\right)$ & $\mathrm{A}(27)$ & $28.4 \pm 0.7$ & $-6.9 \pm 1.2$ & $9.5 \pm 1.2$ & $5.09 \pm 0.04$ \\
\hline & & $O\left(p^{3}\right)$ & $\mathrm{A}(35)$ & $28.3 \pm 0.7$ & $-6.5 \pm 1.0$ & $9.8 \pm 1.0$ & $5.09 \pm 0.04$ \\
\hline & & $O\left(p^{3}\right)$ & $\mathrm{B}(11)$ & $28.3 \pm 1.0$ & $-18.2 \pm 3.4$ & $-1.9 \pm 3.4$ & $5.09 \pm 0.04$ \\
\hline & & $O\left(p^{3}\right)$ & $\mathrm{B}(16)$ & $28.8 \pm 0.9$ & $-17.3 \pm 2.0$ & $-0.9 \pm 2.0$ & $5.09 \pm 0.04$ \\
\hline & & $O\left(p^{3}\right)$ & $\mathrm{B}(27)$ & $28.4 \pm 0.7$ & $-18.6 \pm 1.2$ & $-2.2 \pm 1.2$ & $5.09 \pm 0.04$ \\
\hline & & $O\left(p^{3}\right)$ & $\mathrm{B}(35)$ & $28.2 \pm 0.7$ & $-19.3 \pm 1.0$ & $-2.9 \pm 1.0$ & $5.09 \pm 0.04$ \\
\hline & Dispersion theory & & & $28.0 \pm 0.2$ & -9.6 & 6.1 & 4.9 \\
\hline
\end{tabular}

and $m_{1-}^{(0)}$ depend only on $b_{10}$. Here the $\mathrm{B}(35)$ solution is clearly ruled out by comparison with the dispersion relation results. The $\mathrm{A}(35)$ solution agrees moderately well, especially since the dispersion results come from taking the difference of two large numbers, and so probably have significant uncertainties. As found before however, the convergence of the magnetic multipoles is not as good as for the electric multipoles.

One can summarize the results of this evaluation of the threshold multipoles and comparison with the dispersion relation calculation of Ref. [8] as follows. Generally the HBChPT calculation produces results for the multipoles for the physical processes that converge and that agree with the dispersion relation calculation. Likewise the various LEC's seem to be well determined. The second solution, $\mathrm{B}(35)$, which could not be distinguished from the other one on the basis of $\chi^{2}$ alone, seems to be ruled out by comparison with dispersion relation results. The weakest link appears to be in the convergence of the HBChPT expansion for the magnetic multipoles, which is not as good as that for the electric multipoles, and in the detailed combinations of multipoles depending on $b_{10}$ alone.

To improve the calculation it might be interesting to extend it to one higher order, which can be done still within the context of a one-loop calculation. Thus one could see if the $O\left(p^{4}\right)$ terms indicate real convergence. One might also think about including the $\Delta(1232)$ as an explicit degree of freedom. In the present calculation $\Delta$ effects are included implicitly in the LEC's, which is a perfectly consistent approach. One alternatively could extract them explicitly along the

TABLE III. Four combinations of $s$ - and $p$-wave threshold multipoles that are independent of all $b_{i}$ parameters (including $\left.b_{19}\right)$ up to and including $O\left(p^{3}\right)$ in HBChPT. Their values are compared to the dispersion theory results of Ref. [8]. The $E_{0+}$ are in units of $10^{-3} / m_{\pi^{+}}$and the reduced $p$-wave multipoles are in units of $10^{-3} / \mathrm{m}_{\pi^{+}}^{3}$.

\begin{tabular}{ccccc}
\hline \hline & & HBChPT & Dispersion theory \\
& $O(p)$ & $O\left(p^{2}\right)$ & $O\left(p^{3}\right)$ & \\
\hline$E_{0+}^{(0)}$ & 0 & -1.5 & -1.4 & $-1.3 \pm 0.1$ \\
$e_{1+}^{(0)}$ & 0 & 0 & 0.02 & 0 \\
$m_{1+}^{(0)}-m_{1-}^{(0)}$ & 0 & 1.3 & 1.3 & -9.6 \\
$2 m_{1+}^{(-)}+m_{1-}^{(-)}$ & 0 & -7.0 & -8.5 & \\
\hline \hline
\end{tabular}


TABLE IV. Some additional combinations of $s$ - and $p$-wave multipoles that depend on subsets of the $b_{i}$ parameters up to and including $O\left(p^{3}\right)$ in HBChPT. Their values are compared to the dispersion theory results of Ref. [8]. The $E_{0+}$ are in units of $10^{-3} / m_{\pi^{+}}$and the reduced $p$-wave multipoles are in units of $10^{-3} / m_{\pi^{+}}^{3}$.

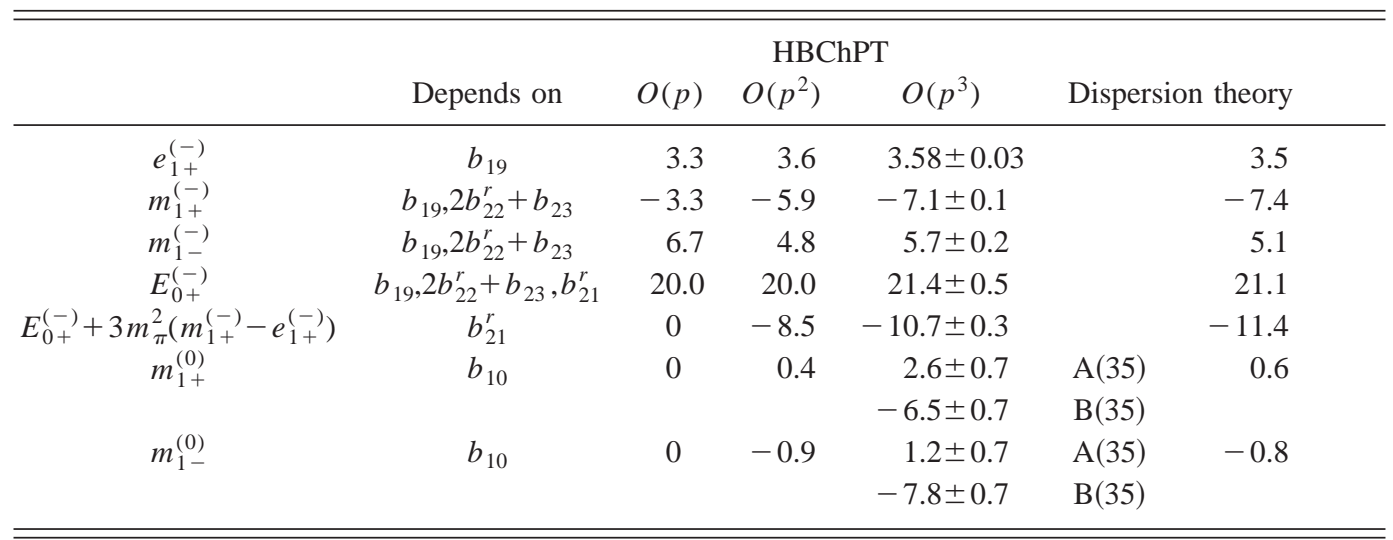

lines of Ref. [4]. Very preliminary estimates seem to indicate that such effects are relatively small in the very near threshold region we are considering, but it might be worth doing a full calculation.

Finally, as somewhat of a side issue, we note that an alternative representation of the near-threshold differential cross section which is often used is

$$
\begin{gathered}
\frac{\omega}{|\vec{q}|} \frac{d \sigma^{\gamma N \rightarrow \pi N}}{d \Omega_{\pi}}=A+B x+C x^{2}, \\
A=\left|E_{0+}\right|^{2}+\frac{1}{2}\left|P_{2}\right|^{2}+\frac{1}{2}\left|P_{3}\right|^{2}, \\
B=2 \operatorname{Re}\left(E_{0+} P_{1}^{*}\right), \\
C=\left|P_{1}^{2}\right|-\frac{1}{2}\left|P_{2}\right|^{2}-\frac{1}{2}\left|P_{3}\right|^{2}, \\
P_{1}=3 E_{1+}+M_{1+}-M_{1-}, \\
P_{2}=3 E_{1+}-M_{1+}+M_{1-}, \\
P_{3}=2 M_{1+}+M_{1-} .
\end{gathered}
$$

However, this near-threshold result differs somewhat from the general result we have used. It is obtained by expanding the original amplitude, e.g., the pion pole contributions, and keeping terms only through $x^{2}$, which is sufficient to give the cross section in terms of $s$ - and $p$-wave multipoles. In contrast we used the square of the full $\mathrm{HBChPT}$ amplitude to get the cross section, and only later after fitting the data extracted the $s$ - and $p$-wave multipoles.

\section{SUMMARY AND OUTLOOK}

We have investigated the radiative capture of a charged pion by a nucleon using heavy baryon chiral perturbation theory and have obtained explicit expressions for the amplitude and for the $s$ - and $p$-wave multipoles, expressed, as is more conventional, as amplitudes for the inverse photoproduction process. Up to $O\left(p^{3}\right)$, these expressions depend upon three parameters that were determined by fitting to data for $\pi^{-}$capture by a proton and for very near threshold photoproduction. Two satisfactory fits were obtained, which were indistinguishable, based only on comparison with the data.

Using the LEC's obtained from these fits, the eight $s$ - and $p$-wave multipoles (four for the $\pi^{+}$case and four for the $\pi^{-}$ case) were calculated and compared with results previously obtained from dispersion theory [8]. In general the agreement was good for one of the fits, A(35), whereas there were significant differences when the other fit was used. This same result held for combinations of the multipoles depending on just a few of the parameters. We thus conclude that the $\mathrm{A}(35)$ fit gives an acceptable result, and thus that the three parameters determined in that fit, $b_{10}, b_{21}^{r}, b_{22}^{r}$ and given in Table I are available for future studies of other reactions.

In general the convergence of the HBChPT expansion was very good for the electric multipoles, but somewhat less good for the magnetic ones. This suggests that it might be valuable to consider extending the present work to $O\left(p^{4}\right)$ or to include explicit $\Delta$ (1232) fields in the chiral Lagrangian.

\section{ACKNOWLEDGMENTS}

We are grateful to Dave Hutcheon for providing us with unpublished TRIUMF data, to Elie Korkmaz for the data from the SAL experiment, and to Dave Hutcheon, Lothar Tiator, and Olaf Hanstein for helpful conversations. This work was supported in part by the Natural Sciences and Engineering Research Council of Canada.

\section{APPENDIX: STRUCTURE AMPLITUDES}

Up to $O\left(p^{3}\right)$ in HBChPT, the structure amplitudes of Eq. (6), corresponding to the photoproduction process $\gamma N \rightarrow \pi N$, are found to be 


$$
\begin{aligned}
& F_{1}^{(0)}\left(E_{\pi}, x\right)=\frac{m_{N}}{4 \pi \sqrt{s}} \frac{e G_{A}}{2 F}\left\{\frac{1}{2 m_{N}}\left[-E_{\pi}+x|\vec{q}|\left(\mu_{p}+\mu_{n}\right)\right]+\frac{2 x E_{\pi}|\vec{q}| b_{10}}{G_{A}(4 \pi F)^{2}}\right. \\
& \left.+\frac{1}{4 m_{N}^{2}}\left[-|\vec{q}|^{2}-\frac{1}{2} x E_{\pi}|\vec{q}|+\left(2 E_{\pi}^{2}-m_{\pi}^{2}+x E_{\pi}|\vec{q}|-2 x^{2}|\vec{q}|^{2}\right)\left(\mu_{p}+\mu_{n}\right)\right]\right\}, \\
& F_{1}^{(-)}\left(E_{\pi}, x\right)=\frac{m_{N}}{4 \pi \sqrt{s}} \frac{e G_{A}}{2 F}\left(1-\frac{x|\vec{q}|}{2 m_{N}}\left(\mu_{p}-\mu_{n}\right)+\frac{1}{4 m_{N}^{2}}\left[E_{\pi}^{2}-\frac{m_{\pi}^{2}}{2}-\left(2 E_{\pi}^{2}-m_{\pi}^{2}+x E_{\pi}|\vec{q}|-2 x^{2}|\vec{q}|^{2}\right)\left(\mu_{p}-\mu_{n}\right)\right]\right. \\
& -\frac{2 m_{\pi}^{2} b_{19}}{G_{A}(4 \pi F)^{2}}+\frac{2 E_{\pi}^{2}}{G_{A}(4 \pi F)^{2}}\left(b_{21}^{r}(\mu)-\frac{G_{A}}{2}\left(1+G_{A}^{2}\right) \ln \frac{m_{\pi}^{2}}{\mu^{2}}\right)+\frac{E_{\pi}\left(E_{\pi}-x|\vec{q}|\right)}{G_{A}(4 \pi F)^{2}}\left(2 b_{22}^{r}(\mu)+b_{23}+G_{A}^{3} \ln \frac{m_{\pi}^{2}}{\mu^{2}}\right) \\
& +\frac{1}{4(4 \pi F)^{2}}\left\{\pi^{2} m_{\pi}^{2}-8 E_{\pi}|\vec{q}| \ln \left(\frac{E_{\pi}+|\vec{q}|}{m_{\pi}}\right)+4 i \pi m_{\pi}^{2} \ln \left(\frac{E_{\pi}+|\vec{q}|}{m_{\pi}}\right)-4 m_{\pi}^{2}\left[\ln \left(\frac{E_{\pi}+|\vec{q}|}{m_{\pi}}\right)\right]^{2}\right. \\
& \left.\left.+4 i \pi E_{\pi}|\vec{q}|\right\}+\frac{x G_{A}^{2} E_{\pi}|\vec{q}|}{(4 \pi F)^{2}}\left[2-\frac{2|\vec{q}|}{E_{\pi}} \ln \left(\frac{E_{\pi}+|\vec{q}|}{m_{\pi}}\right)+\frac{\pi^{2} m_{\pi}^{2}}{4 E_{\pi}^{2}}-\frac{2 \pi m_{\pi}}{E_{\pi}}+\frac{m_{\pi}^{2}}{E_{\pi}^{2}}\left[\ln \left(\frac{E_{\pi}+|\vec{q}|}{m_{\pi}}\right)\right]^{2}\right]\right) \\
& F_{2}^{(0)}\left(E_{\pi}, x\right)=\frac{m_{N}}{4 \pi \sqrt{s}} \frac{e G_{A}}{2 F}\left\{\frac{E_{\pi}|\vec{q}|}{8 m_{N}^{2}}-\frac{x|\vec{q}|^{2}}{4 m_{N}^{2}}\left(\mu_{p}+\mu_{n}\right)+\frac{2 E_{\pi}|\vec{q}| b_{10}}{G_{A}(4 \pi F)^{2}}\right\} \\
& F_{2}^{(-)}\left(E_{\pi}, x\right)=\frac{m_{N}}{4 \pi \sqrt{s}} \frac{e G_{A}}{2 F}\left\{-\frac{|\vec{q}|}{2 m_{N}}\left(\mu_{p}-\mu_{n}\right)+\frac{|\vec{q}|}{4 m_{N}^{2}}\left[E_{\pi}-\left(E_{\pi}-x|\vec{q}|\right)\left(\mu_{p}-\mu_{n}\right)\right]\right. \\
& \left.+\frac{G_{A}^{2} E_{\pi}|\vec{q}|}{2(4 \pi F)^{2}}\left[\frac{\pi^{2} m_{\pi}^{2}}{E_{\pi}^{2}}-\frac{4 \pi m_{\pi}}{E_{\pi}}-2 \pi i \frac{|\vec{q}|}{E_{\pi}}+2 \pi i \frac{m_{\pi}^{2}}{E_{\pi}^{2}} \ln \left(\frac{E_{\pi}+|\vec{q}|}{m_{\pi}}\right)\right]\right\} \\
& F_{3}^{(0)}\left(E_{\pi}, x\right)=\frac{m_{N}}{4 \pi \sqrt{s}} \frac{e G_{A}}{2 F}\left\{-\frac{|\vec{q}|}{2 m_{N}}\left(\mu_{p}+\mu_{n}\right)+\frac{E_{\pi}|\vec{q}|}{8 m_{N}^{2}}\left[3-2\left(\mu_{p}+\mu_{n}\right)\right]+\frac{x|\vec{q}|^{2}}{2 m_{N}^{2}}\left(\mu_{p}+\mu_{n}\right)-\frac{2 E_{\pi}|\vec{q}| b_{10}}{G_{A}(4 \pi F)^{2}}\right\} \\
& F_{3}^{(-)}\left(E_{\pi}, x\right)=\frac{m_{N}}{4 \pi \sqrt{s}} \frac{e G_{A}}{2 F}\left(\frac{|\vec{q}|}{\left(E_{\pi}-x|\vec{q}|\right)}+\frac{|\vec{q}|}{2 m_{N}}\left(\mu_{p}-\mu_{n}\right)+\frac{|\vec{q}|}{m_{N}^{2}}\left[-\frac{E_{\pi}}{4}-\frac{m_{\pi}^{2}}{8\left(E_{\pi}-x|\vec{q}|\right)}\right.\right. \\
& \left.+\frac{1}{4}\left(E_{\pi}-2 x|\vec{q}|\right)\left(\mu_{p}-\mu_{n}\right)\right]-\frac{2 m_{\pi}^{2}|\vec{q}| b_{19}}{G_{A}(4 \pi F)^{2}\left(E_{\pi}-x|\vec{q}|\right)}+\frac{E_{\pi}|\vec{q}|}{G_{A}(4 \pi F)^{2}}\left(2 b_{22}^{r}(\mu)+b_{23}+G_{A}^{3} \ln \frac{m_{\pi}^{2}}{\mu^{2}}\right) \\
& \left.+\frac{G_{A}^{2} E_{\pi}|\vec{q}|}{(4 \pi F)^{2}}\left\{-2+\frac{2|\vec{q}|}{E_{\pi}} \ln \left(\frac{E_{\pi}+|\vec{q}|}{m_{\pi}}\right)-\frac{\pi^{2} m_{\pi}^{2}}{4 E_{\pi}^{2}}-\frac{m_{\pi}^{2}}{E_{\pi}^{2}}\left[\ln \left(\frac{E_{\pi}+|\vec{q}|}{m_{\pi}}\right)\right]^{2}+\frac{2 \pi m_{\pi}}{E_{\pi}}\right\}\right), \\
& F_{4}^{(0)}\left(E_{\pi}, x\right)=\frac{m_{N}}{4 \pi \sqrt{s}} \frac{e G_{A}}{2 F}\left\{\frac{|\vec{q}|^{2}}{2 m_{N} E_{\pi}}+\frac{1}{m_{N}^{2}}\left[\frac{E_{\pi}^{2}}{4}-\frac{m_{\pi}^{4}}{4 E_{\pi}^{2}}-\frac{x|\vec{q}|^{3}}{2 E_{\pi}}-\frac{|\vec{q}|^{2}}{4}\left(\mu_{p}+\mu_{n}\right)\right]\right\}, \\
& F_{4}^{(-)}\left(E_{\pi}, x\right)=\frac{m_{N}}{4 \pi \sqrt{s}} \frac{e G_{A}}{2 F}\left\{-\frac{|\vec{q}|^{2}}{E_{\pi}\left(E_{\pi}-x|\vec{q}|\right)}-\frac{|\vec{q}|^{2}}{2 m_{N} E_{\pi}}\left[1+\frac{m_{\pi}^{2}}{E_{\pi}\left(E_{\pi}-x|\vec{q}|\right)}\right]\right. \\
& \left.-\frac{|\vec{q}|^{2}}{4 m_{N}^{2}}\left[1+\frac{m_{\pi}^{2}}{E_{\pi}^{2}}-\frac{2 x|\vec{q}|}{E_{\pi}}-\left(\mu_{p}-\mu_{n}\right)-\frac{3 m_{\pi}^{2}}{2 E_{\pi}\left(E_{\pi}-x|\vec{q}|\right)}+\frac{m_{\pi}^{4}}{E_{\pi}^{3}\left(E_{\pi}-x|\vec{q}|\right)}\right]+\frac{2 m_{\pi}^{2}|\vec{q}|^{2} b_{19}}{G_{A}(4 \pi F)^{2} E_{\pi}\left(E_{\pi}-x|\vec{q}|\right)}\right\}
\end{aligned}
$$


where $|\vec{q}|=\sqrt{E_{\pi}^{2}-m_{\pi}^{2}}, m_{N}$ is the renormalized nucleon mass, and $m_{\pi}$ is the renormalized pion mass.

Note that all of the parameters in these expressions have been renormalized. The calculation was performed using the bare Lagrangian parameters, which were then converted to renormalized parameters as follows:

$$
\begin{gathered}
2 a_{7}=\mu_{p}+\mu_{n}, \\
4 a_{6}=\mu_{p}-\mu_{n}+\frac{4 \pi G_{A}^{2} m_{\pi} m_{N}}{(4 \pi F)^{2}}, \\
F_{0}=F\left\{1-\frac{m_{\pi}^{2}}{F^{2}}\left[l_{4}^{r}(\mu)-\frac{1}{(4 \pi)^{2}} \ln \left(\frac{m_{\pi}^{2}}{\mu^{2}}\right)\right]\right\},
\end{gathered}
$$

$$
\begin{aligned}
g_{A}= & G_{A}-\frac{4 a_{3} G_{A} m_{\pi}^{2}}{m_{N}^{2}}+\frac{G_{A}^{3} m_{\pi}^{2}}{(4 \pi F)^{2}}-\frac{4 m_{\pi}^{2}}{(4 \pi F)^{2}} \\
& \times\left[b_{17}^{r}(\mu)-\frac{G_{A}}{4}\left(1+2 G_{A}^{2}\right) \ln \left(\frac{m_{\pi}^{2}}{\mu^{2}}\right)\right] .
\end{aligned}
$$

$\mu_{p} \simeq 2.79$ and $\mu_{n} \simeq-1.91$ are the magnetic moments of the proton and neutron, respectively. The expression for the bare pion decay constant $F_{0}$ in terms of the renormalized $F$ and for the bare $g_{A}$ in terms of the physical $G_{A} \simeq 1.26$ depend somewhat on the explicit form of the Lagrangian used, and are derived, for example, in Ref. [12].
[1] V. Bernard, N. Kaiser, and U.-G. Meißner, Phys. Lett. B 383, 116 (1996).

[2] V. Bernard, N. Kaiser, and U.-G. Meißner, Z. Phys. C 70, 483 (1996).

[3] H. W. Fearing, R. Lewis, N. Mobed, and S. Scherer, Nucl. Phys. A631, 735c (1998); T. Meissner, F. Myhrer, and K. Kubodera, Phys. Lett. B 416, 36 (1998); S.-I. Ando and D.-P. Min, ibid. 417, 177 (1998); V. Bernard, T. R. Hemmert, and U.-G. Mei/ßner, hep-ph/9811336; nucl-th/0001052.

[4] T. R. Hemmert, B. R. Holstein, and J. Kambor, Phys. Lett. B 395, 89 (1997); J. Phys. G 24, 1831 (1998); T. R. Hemmert et al., Phys. Rev. D 57, 5746 (1998).

[5] M. Salomon, D. F. Measday, J.-M. Poutissou, and B. C. Rob- ertson, Nucl. Phys. A414, 493 (1984).

[6] D. Hutcheon (private communication); Kailin Liu, Ph.D. thesis, University of Kentucky, 1994.

[7] E. Korkmaz et al., Phys. Rev. Lett. 83, 3609 (1999).

[8] O. Hanstein, D. Drechsel, and L. Tiator, Phys. Lett. B 399, 13 (1997); Nucl. Phys. A632, 561 (1998).

[9] G. F. Chew, M. L. Goldberger, F. E. Low, and Y. Nambu, Phys. Rev. 106, 1345 (1957).

[10] F. A. Berends, A. Donnachie, and D. L. Weaver, Nucl. Phys. B4, 1 (1967).

[11] G. Ecker and M. Mojžišs, Phys. Lett. B 365, 312 (1996).

[12] H. W. Fearing, R. Lewis, N. Mobed, and S. Scherer, Phys. Rev. D 56, 1783 (1997). 